\title{
Is Barbero's Hamiltonian formulation a Gauge Theory of Lorentzian Gravity?
}

\author{
Joseph Samuel \\ Raman Research Institute \\ Bangalore 560 080, INDIA.
}

email:sam@rri.ernet.in 


\begin{abstract}
This letter is a critique of Barbero's constrained Hamiltonian formulation of General Relativity on which current work in Loop Quantum Gravity is based. While we do not dispute the correctness of Barbero's formulation of general relativity, we offer some criticisms of an aesthetic nature. We point out that unlike Ashtekar's complex $S U(2)$ connection, Barbero's real $S O(3)$ connection does not admit an interpretation as a space-time gauge field. We show that if one tries to interpret Barbero's real $S O(3)$ connection as a spacetime gauge field, the theory is not diffeomorphism invariant. We conclude that Barbero's formulation is not a gauge theory of gravity in the sense that Ashtekar's Hamiltonian formulation is. The advantages of Barbero's real connection formulation have been bought at the price of giving up the description of gravity as a gauge field.
\end{abstract}




\section{Introduction}

In the eighties, Ashtekar [1] introduced complex "new variables" on the phase space of General Relativity, which greatly simplified the form of the constraints. These variables are a (densitised) soldering form and a complex $S U(2)$ connection. Ashtekar's motivation was to formulate General Relativity in a manner similar to Yang-Mills fields, so that ideas and techniques used in quantising gauge theories could be imported into Quantum Gravity. This motivation was an attractive one, since the "gauge" description of Nature is a unifying idea that seems to permeate diverse branches of physics. Ashtekar suggested the use of a gauge field ("the connection representation") as the basic configuration variable in canonical gravity instead of the more traditional "metric representation". This area has been an active one [2]. Witten's paper [3] on the solution of $2+1$ gravity was partly inspired by Ashtekar's suggestion. In $2+1$ gravity the connection representation [3, 4, 5] has proved extremely useful and yields a considerably simpler formulation of quantum gravity than the metrical description. In both $2+1$ and in $3+1$ dimensions, the connection variable is the pull-back of a space-time connection to a spatial slice $\mathcal{S}$. However, in $3+1$ dimensions, progress has been hampered by the 'reality conditions', which are necessary because of the use of complex variables on the real phase space of General Relativity.

In 1994, Barbero wrote a very influential paper [6] pointing out that a small modification of Ashtekar's original canonical transformation leads to a new Hamiltonian formulation of General Relativity in which the basic variables are t a real $S U(2)$ connection \and a real densitised triad. The form of the Hamiltonian constraint in Barbero's formulation is not as simple as in Ashtekar's original formulation. But this is a small price to pay. There are significant advantages in using real variables on the phase space of General Relativity, because the 'reality conditions' which had to be imposed in Ashtekar's original formulation, are no longer necessary. As a result, Barbero's Hamiltonian formulation (BHF) has gained wide acceptance and is currently the basis of Loop Quantum Gravity. A lot of work [7] has been done on the space of real $S U(2)$ connections on manifolds. Since $S U(2)$ is a compact group it has an invariant (Haar) measure, and one is able to achieve a high degree of mathematical control over the space of connections.

\footnotetext{
1 These real variables were earlier considered by Ashtekar and discarded in favour of the complex ("new") variables of [1] because the latter simplified the constraints.

${ }^{2}$ Or equivalently $S O(3)$; we will not make this relatively fine distinction in this paper.
} 
It was pointed out by Immirzi [8] that Barbero's canonical transformation could be slightly generalised: a one parameter family of canonical transformations were possible and all of them led to a Hamiltonian formulation based on a real $S U(2)$ connection. This free parameter $\beta$ is known as the "Immirzi parameter", and does not appear to be fixed by any theoretical considerations.

There are several puzzling features about Barbero's Hamiltonian formulation. Viewed as a gauge theory (and this was Ashtekar's original motivation), the gauge group of General Relativity is certainly non-compact. Depending on the approach, one might either believe the gauge group to be the Lorentz group [9] or the Poincare group [10]. It is generally agreed that the gauge group of General Relativity must be non-compact. How then is it possible to formulate GR as gauge theory of a compact group, as Barbero seems to? Of course, it is possible to formulate GR as a gauge theory of a complex $\mathrm{SU}(2)$ group as in the original Ashtekar formulation. Over the complex numbers, there is no distinction between compact and non-compact gauge groups. The puzzle is that Barbero's Hamiltonian formulation uses a gauge group which is both real and compact. Since BHF was derived by a canonical transformation from a diffeomorphism invariant theory, it must of course be diffeomorphism invariant. However, there does not presently exist any manifestly covariant Lagrangian formulation of General Relativity as a gauge theory in which the gauge group is real and compact. How does one understand this?

Holst [1] has given a Lagrangian formulation equivalent to General Relativity and shown that on Legendre transformation, it results in BHF. A curious feature of Holst's derivation is that he starts with a gauge theoretic formulation with a non-compact $S O(3,1)$ gauge group. Yet he is able to make contact with Barbero's formulation, which is based on an $S O(3)$ gauge group. It is not clear a priori how the reduction in the gauge group takes place.

Another puzzling feature of Barbero's Hamiltonian formulation is that there are many of them. For every nonzero, real value of the Immirzi parameter $\beta$ there is a Hamiltonian formulation of General Relativity with as much claim to validity as Barbero's original formulation. It is not easy to understand the origin of the 'Immirzi ambiguity'. It first appears as a parameter in a canonical transformation that one performs on the phase space of GR. However, instead of dissappearing from the final results of the theory (as one might have hoped), it appears in the spectrum of operators and also in the final expression for Black Hole Entropy as calculated using Loop Quantum Gravity [12]. If 
the Immirzi parameter is indeed present in Quantum Gravity, it would seem that a new fundamental constant (which can only be fixed by experiment) has entered into physics. Such quantization ambiguities do occur in other areas of physics (e.g $\theta$ vacua), but they are well understood to arise from a multiply connected configuration space. The Immirzi ambiguity does not appear to be of topological origin and does require understanding.

We emphasize that we do not question the correctness of BHF as a Hamiltonian formulation or its equivalence of BHF to General Relativity. This equivalence is assured since BHF was derived from the ADM formulation of GR by a canonical transformation. The aim of this paper is to understand the basis of BHF as a reformulation of General Relativity. Is BHF a gauge theory of gravity? How does it happen that the gauge group is both real and compact? How does the diffeomorphism invariance of BHF jive with the absence of any manifestly covariant Lagrangian for GR as a real $S O(3)$ gauge theory. In this paper we will answer these questions and thereby clarify some aspects of Barbero's formulation.

Barbero's Hamiltonian formulation can be derived by making a canonical transformation starting from a constrained Hamiltonian Formulation (CHF) due to Ashtekar- the extended phase space construction (EPS). We note in passing that the EPS was an intermediate step in Ashtekar's original derivation of the new variables. The EPS consists of the following ingredients: the basic variables are $\left(\tilde{E}_{i}^{a}, K_{a}^{i}\right)$, which form a canonically conjugate pair. The space-time meaning of these variables is that $\tilde{E}_{i}^{a}$ is a densitised triad on a spatial slice $\mathcal{S}$ and $K_{a}^{i}$ is the extrinsic curvature tensor of $\mathcal{S}$ with one index converted into a triad index. The three metric tensor $q_{a b}$ of $\mathcal{S}$ is a derived object, which can be constructed from $\tilde{E}_{i}^{a}$ as explained in 13. Similarly, the extrinsic curvature of $\mathcal{S}$ can also be expressed in terms of the basic fields. The constraints of the theory are

$$
\begin{aligned}
\epsilon_{i j k} K_{a}^{j} \tilde{E}^{a k} & \approx 0 \\
D_{a}\left[\tilde{E}_{k}^{a} K_{b}^{k}-\delta_{b}^{a} \tilde{E}_{k}^{c} K_{c}^{k}\right] & \approx 0 \\
\sqrt{q} R+\frac{2}{\sqrt{q}} \tilde{E}_{i}^{[a} \tilde{E}_{j}^{b]} K_{a}^{i} K_{b}^{j} & \approx 0,
\end{aligned}
$$

where $D_{a}$ is the covariant derivative associated with $q_{a b}$ and $R$, its scalar curvature. The Hamiltonian is a combination of constraints. (We assume throughout this paper that space is closed so that we can drop spatial boundary terms.) The EPS formulation is [14] strongly diffeomorphism invariant $(\bmod S O(3)$ gauge) with the above space-time interpretation for the basic variables $\left(\tilde{E}_{i}^{a}, K_{a}^{i}\right)$. We recall 14 that a Hamiltonian theory is 
strongly diffeomorphism invariant (SDI) if a) there are constraints whose brackets reflect the Lie algebra of the diffeomorphism group. b) the basic variables transform as is expected of them from their space-time interpretation. For this criterion to be applied, one must first declare the space-time interpretation of the basic variables in the theory.

BHF differs from the EPS only by a canonical transformation. The basic variables in BHF are $\left(\tilde{E}_{i}^{a}, A_{a}^{i}\right)$, where $\tilde{E}_{i}^{a}$ is the same as before and

$$
A_{a}^{i}:=\Gamma_{a}^{i}+\beta K_{a}^{i}
$$

where $\beta$ is the Immirzi parameter and $\Gamma_{a}^{i}$ are the triad spin coefficients. The constraints of the theory are now

$$
\begin{aligned}
\mathcal{D}_{a} \tilde{E}_{i}^{a} & \approx 0 \\
\tilde{E}_{i}^{b} F_{a b}^{i} & \approx 0 \\
\epsilon^{i j k} \tilde{E}_{i}^{a} \tilde{E}_{j}^{b} F_{a b k}-2 \frac{\left(1+\beta^{2}\right)}{\beta^{2}} \tilde{E}_{[i}^{a} \tilde{E}_{j]}^{b}\left(A_{a}^{i}-\Gamma_{a}^{i}\right)\left(A_{b}^{j}-\Gamma_{b}^{j}\right) & \approx 0,
\end{aligned}
$$

where $\mathcal{D}$ is the covariant derivative associated with the Barbero connection (11). Note that the Scalar constraint (4) explicitly contains the Immirzi parameter. If one now endows $A_{a}^{i}$ with a space-time interpretation coming from (1) as a linear combination of the triad spin coefficients of $\mathcal{S}$ and the extrinsic curvature of $\mathcal{S}$, it is clear that the new formulation is still SDI ( $\bmod S O(3)$ gauge), since the starting point was.

The original Ashtekar variables were derived by making the canonical transformation (1) with $\beta=i$ (or equivalently $-i$ ). This choice is distinguished in several ways.

Why $\beta=i$ is special:

1. Simplification of the Hamiltonian constraint: The expression (44) for the Hamiltonian constraint in BHF contains a messy term multiplied by $\left(1+\beta^{2}\right)$. For $\beta= \pm i$ this term disappears and we get a simplified form for the Hamiltonian constraint.

2. Full local Lorentz invariance: As emphasized by Immirzi [8] the time gauge is not needed to derive the original Ashtekar variables. They can be derived from a manifestly covariant action 15 maintaining full local Lorentz invariance. It follows that the theory is manifestly SDI, (i.e., SDI and not just modulo gauge).

3. Space-time interpretation for the connection: The Ashtekar connection (as defined by (1) with $\beta=i$ ) can also be interpreted as a space-time connection. More precisely, 
the Ashtekar connection is the pull back to $\mathcal{S}$ of a space-time connection 1-form. This is evident because Ashtekar's formulation can be derived from a manifestly covariant Lagrangian [15] in which one of the basic fields is an $S L\left(2, \mathbb{C}^{\prime}\right)$ space-time connection. The Ashtekar connection then appears as the pull-back of the space-time $S L(2, \mathbb{C})$ connection to $\mathcal{S}$. Thus we would regard Ashtekar's Hamiltonian Formulation (AHF) as a gauge theoretic reformulation of General Relativity.

For values of $\beta$ other than $\pm i$ these three properties do not obtain.

1. As Barbero [6] points out, the form of the Hamiltonian constraint is more complicated for $\beta$ real, but one can choose to accept it.

2. It appears that one must choose the "time gauge" in order to arrive at Barbero's formulation. In this formulation the full local Lorentz invariance of the Ashtekar formulation is lost. One can also choose to live with this, since this is only a choice of gauge.

3. The Barbero connection does not have a space-time interpretation as the pull back of a space-time connection 1 -form to $\mathcal{S}$. In the case $\beta=i$, one had the option of interpreting the $A_{a}^{i}$ defined by (11) as a space-time connection. If one attempts to do this for real $\beta$, one finds that the theory violates Strong Diffeomorphism Invariance. Under diffeomorphisms tangential to $\mathcal{S}$, the $A_{a}^{i}$ defined by (1) does transform like a connection 1-form. However, for diffeomorphisms that are normal to $\mathcal{S}$, the canonical transformation properties of the $A_{a}^{i}$ do not reflect its proposed space-time interpretation as the pullback of a space-time connection 1-form. This point is explained and proved below.

Claim: Barbero's connection cannot be interpreted as a space-time connection

Proof: Consider a solution $(\mathcal{M}, g)$ of Einstein's equations and a loop $\gamma$ in $\mathcal{M}$. If a connection transforms correctly under space-time diffeomorphisms (i.e, as a 1-form), the trace of the Holonomy of the connection along $\gamma$ should depend only on the loop $\gamma$ and should not depend on the slicing of $\mathcal{M}$. It is easy to construct an example of an empty space solution to Einstein's equations in which the Holonomy of the Barbero Connection changes from being trivial for one slice containing $\gamma$ to non -trivial for another slice containing $\gamma$. Consider flat space-time $(\mathcal{M}, \eta)$ with standard Minkowski coordinates $(t, x, y, z)$ and a loop $\gamma$ which is a circle of radius $R$ described by $t=\sqrt{1+R^{2}}, z=0, x=R \cos \theta, y=R \sin \theta, 0 \leq \theta \leq 2 \pi$. 
The flat slice $\mathcal{S}_{1}$ defined by $t=\sqrt{1+R^{2}}$ contains $\gamma$. Since both the intrinsic and extrinsic curvature of $\mathcal{S}_{1}$ vanish, the holonomy of the Barbero connection is the identity and its trace is 3 . However, the same loop is also contained in the hyperbolic slice, $\mathcal{S}_{2}$ defined by $t^{2}-x^{2}-y^{2}=1$. We now compute the trace of the Holonomy $\operatorname{tr} H(A)$ of the Barbero connection along the same loop $\gamma$.

At the point $y^{\mu}=(t=1, \vec{x}=0) \in \mathcal{S}_{2}$ the standard orthonormal frame $\hat{e}^{I} \mu=\delta^{I} \mu$ ( $I=0,1,2,3$ is a frame index and $\mu=0,1,2,3$ is a space-time index) has the property that $\hat{e}^{0}{ }_{\mu}$ is normal to $\mathcal{S}_{2}$. Let us move this frame to other points $x^{\mu} \in \mathcal{S}_{2}$ by the Lorentz transformation

$$
e^{I}{ }_{\mu}(x):=\Lambda_{\mu}^{\nu}(x) \hat{e}_{\nu}^{I}
$$

where

$$
\Lambda_{\mu}^{\nu}:=\delta_{\mu}^{\nu}+(1-x \cdot y)^{-1}\left(x_{\mu}+y_{\mu}\right)\left(x^{\nu}+y^{\nu}\right)-2 x_{\mu} y^{\nu}
$$

$\Lambda_{\mu}{ }^{\nu}$ has the property that $x_{\mu}=\Lambda_{\mu}{ }^{\nu} y_{\nu}$, which ensures that $e^{0}{ }_{\mu}$ is normal to $\mathcal{S}_{2}$ everywhere. (Our frame satisfies the 'time gauge'.) The space-time connection 1-form is given by $A_{\alpha}{ }^{I J}$

$$
A_{\alpha}^{I J}:=e_{\mu}^{I} \partial_{\alpha} e^{\mu J}
$$

( $A^{I J}$ with two internal indices is the space-time connection and should not be confused with the Barbero connection $A^{i}$, which has one internal index.) The contraction $A^{I J}:=t^{\alpha} A_{\alpha}$ of the connection one-form with the tangent vector $t^{\alpha}$ to the curve $\gamma$ is easily worked out. The non-vanishing components are $A^{01}=-A^{10}=-y, A^{02}=-A^{20}=x, A^{12}=-A^{21}=1-t$ Constructing Barbero's connection by the formula

$$
A^{i}=1 / 2 \epsilon^{i j k} A^{j k}+\beta A^{0 i}
$$

we find that $A^{1}=-\beta y, A^{2}=\beta x, A^{3}=1-t$. An elementary calculation then yields the result

$$
\operatorname{Tr} H(A)=1+2 \cos \left(2 \pi \sqrt{1+R^{2}\left(1+\beta^{2}\right)}\right)
$$

Notice that the trace of the Holonomy of the Barbero connection along the same loop $\gamma$, depends on $\beta$ and (except for the special values $\beta= \pm i$ ) is not the equal to 3 . Briefly, the trace of the holonomy of the Barbero Connection along a loop $\gamma$ is not just a property of the loop but also depends on the slicing.

What this means is that it is impossible to attach a gauge theoretic space-time interpretation to Barbero's connection. As a spatial connection, Barbero's connection is 
certainly well defined and, in fact, transforms correctly under diffeomorphisms that preserve the spatial slice. But unlike Ashtekar's connection, Barbero's connection does not admit an interpretation as a space-time gauge field. Such an interpretation is not consistent with the Poisson bracket relations between $A_{a}^{i}$ and the Scalar constraint. It appears that the "extra" term proportional to $1+\beta^{2}$ ruins this bracket. Thus BHF is not a gauge theory of gravitation.

This observation removes most of the puzzles we had raised at the beginning of this letter. BHF is not a gauge theory of gravitation and so one cannot identify Barbero's $S O(3)$ with the gauge group of gravity. There is no conflict between Barbero's Hamiltonian formulation and our expectation that the gauge group of gravity must be noncompact.

This observation also removes the puzzle of how Holst is able to derive a compact gauge group from a non compact one. The variable defined as a connection variable by Holst [1]] is not the pullback of a space-time connection. Rather, certain components of the space-time connection are defined to be components of a new $S O(3)$ connection. This is Barbero's connection and it has no space-time significance. One sometimes sees it implied [8, 11] that the reduction in the gauge group from $S O(3,1)$ to $S O(3)$ takes place because of our choice of the "time gauge". It is indeed true that once we make this gauge choice ${ }^{3}$, our freedom to make additional gauge transformations is curtailed from $\operatorname{SO}(3,1)$ to $S O(3)$. However this does not mean that the gauge group has been reduced. The pullback of the connection to a spatial slice is still an $S O(3,1)$ connection, in spite of our gauge choice.

While this point is elementary, it is worth making in detail and we digress briefly to do so. The central point here is a geometrical notion: the holonomy group of a connection [16]. Given a $G$ connection $A$ on a manifold $\mathcal{S}$, we define its holonomy group to be the collection $H_{p}(A)=\left\{H_{\gamma_{p}}(A)\right\}$ of elements of $G$ which arise as holonomies of $A$ along closed loops $\gamma_{p}$ based at $p$. For example, if the connection is pure gauge, the holonomy group is trivial. It is easily seen that the holonomy groups based at $p$ and $p^{\prime} \in \mathcal{S}$ are related by conjugation and therefore isomorphic. Further, under a local gauge transformation, the holonomy group of $A$ transforms by conjugation

$$
H_{p}(A) \rightarrow g(p) H_{p}(A) g^{-1}(p)
$$

${ }^{3}$ Holst's orginal derivation of BHF from his covariant Lagrangian contained a small logical gap: he used the time gauge fixed action to perform the Legendre transformation. This gap has since been filled in papers by S. Alexandrov 17] and Nuno Barros e Sa 18] 
where $g(p) \in G$. We say that a connection $A$ is reducible if its holonomy group is a proper subgroup of $G$. Clearly, the reducibility of a connection is a gauge invariant notion and is independent of gauge choice. A general $S O(3,1)$ connection is not reducible to $S O(3)$.

One may of course, simply abandon the "gauge interpretation" of the theory and view the Barbero connection as a purely spatial connection which one uses for technical reasons to produce quantum states which are functionals of the extrinsic curvature. But then one must be aware that one has given up the gauge interpretation. We argue here that there are strong aesthetic reasons for retaining the gauge interpretation. One of the principal motivations of the Ashtekar program was the gauge description of gravity. This appears to be a unifying thread across the different forces of nature: they are all described by a space-time gauge field. The view we would like to offer here is that this was an important motivation of the original Ashtekar program and should be retained. From this point of view, the Immirzi parameter is not a free parameter but must be fixed to the special value $i$. This is one possible resolution of the "Immirzi Ambiguity". Similar views have been expressed (see footnote 3 of [17) by Alexandrov [17.

If one gives up the gauge interpretation of gravity, the Immirzi parameter appears not to be fixed by theory. This would not be a problem if the parameter dissappeared from all physical predictions of the theory. However, this is not the case: the Immirzi parameter does appear in the calculated value of Black Hole entropy in Loop Quantum Gravity. This phenomenon does not appear to be well understood (unlike the $\theta$ vacua of QCD). Rovelli and Thiemann [19] offer a finite dimensional example of the Immirzi ambiguity. We do not find this example convincing: their original system does not suffer from any ambiguity. The ambiguity is introduced 'by hand' by changing the original configuration space in a $\beta$ dependent manner. Thus their "quantisation prodedure" does not quantise the original system at all, but quantises a one parameter family of distinct systems [20].

If one accepts the idea that canonical transformations made by the theorist can introduce parameters into the physical predictions of the quantum theory, one does lose predictive power. Admittedly, this loss is very small in the case of the Immirzi ambiguity. The ambiguity is to the extent of a single parameter which can be fixed by comparing a single prediction of the theory with an independent calculation (say the Black Hole entropy) or (thinking wishfully) an experiment. However, we would argue that in a field

\footnotetext{
${ }^{4}$ as in Alexandrov's path integral quantisation [17.
} 
theory like General Relativity, there are infinitely many degrees of freedom (two at each spatial point) and one could, in principle, contemplate making separate canonical transformations in each of them, thereby introducing an enormous ambiguity into the theory. In the absence of any internal criterion to curb this ambiguity, the physical predictions of the theory may depend on an infinite number of parameters. Such a theory would have no predictive power.

If one wishes to describe Euclidean Gravity rather than Lorentzian Gravity, the gauge group is automatically compact and the Ashtekar variables are real. Barbero's connection (with $\beta=1$ ) agrees with Ashtekar's and one does have a real formulation of Euclidean gravity as a gauge theory. However, for $\beta$ not equal to unity, the same problem arises: Barbero's connection is not a space-time connection and if one attempts to interpret it as such, one violates diffeomorphism invariance. It appears then that the values $\beta=1$ (for Euclidean) and $\beta=i$ (for Lorentzian Gravity) are very special. One could hope to relate these two by a Wick rotation as discussed in 21]

In this paper we argue strongly for maintaining the gauge aspect of gravity in the approach to quantum gravity, even though the gauge group is non-compact and therefore not as tractable as say, $S U(2)$. It does not appear to us a strong argument to say that we study compact gauge groups because we do not know how to deal with non-compact gauge groups with mathematical rigour. The non-compactness of the gauge group appears to us an essentially physical feature of General Relativity, which is closely related to the Minkowskian signature of the space-time metric and light cones. Phenomena like infinite red shift, seen in Black Hole physics need non-compact groups for a gauge theoretical description. We would suggest that one must learn to deal with non-compact gauge groups. One could return either to the original Ashtekar variables with a complex connection or to the real Palatini tetrad formulation, with non-compact gauge group $S O(3,1)$. In our opinion these two problems - non-compact gauge groups and complex gauge groups- are the same problem in different guises. Evading both problems simultaneously is impossible, if one is interested in dealing with the physical effects of Lorentzian General Relativity as a gauge theory.

Acknowledgement: It is a pleasure to thank Richard Epp, B.R. Iyer, Sukanya Sinha and Madhavan Varadarajan for extended discussions and Ramesh Anishetty, Ghanashyam Date, N.D. Haridass, T.R. Govindrajan, Romesh Kaul and H.S Sharatchandra for their critical comments on this work. 


\section{References}

[1] Ashtekar A (1986) Phys. Rev. Lett. 57 2244; Ashtekar A (1987) Phys. Rev. D36 1587; see also [13].

[2] For exhaustive references in this area, see the review article by Gaul, $\mathrm{M}$ and Rovelli C, grqc/99100 79.

[3] Witten E (1998) Nucl. Phys. B311 46.

[4] Achucarro A and Townsend P K (1986) Phys. Lett. B180 89.

[5] Carlip S (1998) Quantum Gravity in 2+1 Dimensions, CUP, Cambridge.

[6] Barbero, J F Phys. Rev. D., 51, 5507 (1995), gr-qc/9410014.

[7] see Rovelli, C. Loop Quantum Gravity, Living Reviews gr-qc 9710008.

[8] Immirzi, G (1997) Class. Quant. Gravity 14, L177-181. gr-qc/9511026.

[9] Utiyama R, 1956 Phys. Rev 1011597.

[10] Kibble T W B, 1961 Journ. Math. Phys. 2212.

[11] Holst S (1996) Phys. Rev. D53, 5966, gr-qc/9511026.

[12] Ashtekar A, Baez J, Corichi A and Krasnov K (1998) Phys. Rev. Lett. 80, 904.

Kaul, R K and Majumdar P (2000), Phys. Rev. Lett (to appear) gr-qc/0002040.

[13] Ashtekar A (notes prepared in collaboration with R. Tate) (1991) Lectures on Non-perturbative canonical gravity, World Scientific, Singapore.

[14] Samuel J, (2000) Canonical Gravity, Diffeomorphisms and Objective Histories preprint.

[15] Jacobson T and Smolin L (1988) Class. and Quant. Grav. 5 583; Samuel J (1987) Pramana-J. Phys. 28 L429.

[16] Cartan E, (1986) 'On Manifolds with an affine connection and the theory of General Relativity', English translation by Anne Magnon and Abhay Ashtekar, Bibliopolis, Naples.

[17] Alexandrov S (2000) "SO $(4, C)$ - covariant Ashtekar-Barbero gravity and the Immirzi parameter", gr-qc/0005085

[18] Barros e Sa N (2000) "Hamiltonian Analysis of General Relativity with the Immirzi Parameter" gr-qc/0006013.

[19] Rovelli C and Thiemann T, The Immirzi parameter in quantum general relativity gr-qd 9705059 .

[20] Samuel J (2000) "Comment on the Immirzi parameter in Quantum General Relativity", submitted to Physical Review .

[21] Ashtekar A (1996) Phys. Rev. D53 2865. 